\title{
A Dual-Wideband Double-Layer Magnetoelectric Dipole Antenna with a Modified Horned Reflector for 2G/3G/LTE Applications
}

\author{
Botao Feng, ${ }^{1}$ Weijun Hong, ${ }^{1}$ Shufang Li, ${ }^{1}$ Wenxing An, ${ }^{2}$ and Sixing Yin ${ }^{1}$ \\ ${ }^{1}$ School of Information and Communication Engineering, Beijing University of Posts and Telecommunications, Beijing 100876, China \\ ${ }^{2}$ Department of Electronic Engineering, School of Information Science and Technology, Tsinghua University, Beijing 100084, China \\ Correspondence should be addressed to Botao Feng; fengbotao@bupt.edu.cn
}

Received 17 July 2013; Revised 5 November 2013; Accepted 10 November 2013

Academic Editor: Zhongxiang Shen

Copyright (C) 2013 Botao Feng et al. This is an open access article distributed under the Creative Commons Attribution License, which permits unrestricted use, distribution, and reproduction in any medium, provided the original work is properly cited.

A novel dual-wideband double-layer magnetoelectric dipole unidirectional antenna with a modified horned reflector for $2 \mathrm{G} / 3 \mathrm{G} / \mathrm{LTE}$ applications is proposed. Firstly, a double-layer electric dipole structure is presented to provide a dualwideband, whose folded lower layer mainly serves the lower frequency band while the inclined upper layer works for the upper frequency band. In addition, to reduce the size of the antenna and improve impedance matching, a new feeding structure designed with inverted Ushaped and tapered line is introduced. Finally, a modified horn-shaped reflector, instead of a ground plane, is employed to achieve stable and high gains. The antenna prototype can achieve a bandwidth of $24.4 \%(790 \mathrm{MHz}-1010 \mathrm{MHz})$ with a stable gain of $7.2 \pm$ $0.6 \mathrm{dBi}$ for the lower band, and a bandwidth of $67.3 \%(1.38 \mathrm{GHz}-2.78 \mathrm{GHz})$ with a gain of $7.5 \pm 0.8 \mathrm{dBi}$ for the upper band covering all the frequency bands for $2 \mathrm{G} / 3 \mathrm{G} / \mathrm{LTE}$ systems. To the best of our knowledge, it is the first double-layer magnetoelectric dipole antenna proposed. Compared with the existing ME dipole antennas, the proposed antenna, which is completely made of copper, can be easily fabricated at low cost and thus is practicable for 2G/3G/LTE applications.

\section{Introduction}

With the rapid development of modern wireless communications, dual-band antennas have been widely applied in 2G/3G/LTE(4G) systems, where two separated frequency bands centered at $900 \mathrm{MHz}(810-960 \mathrm{MHz})$ and $2.2 \mathrm{GHz}$ $(1.71-2.69 \mathrm{GHz})$ have been allocated in China [1]. In virtue of their advantages such as reducing numbers of antennas and minimizing installation area, the dual-band antennas have attracted tremendous attention. On the other hand, recently, there is an increasing interest in magnetoelectric (ME) dipole antennas, which can meet the high requirement of wideband mobile communications due to their excellent electrical characteristics, such as low cross-polarization, low back-lobe radiation, stable gain across the operating band and nearly identical $E$ - and $H$-plane patterns. With staircaseshaped feeding strip [2-6], differential feeding structure [7, 8 ], or special dielectric material [8-10], this kind of antenna is able to achieve outstanding electrical performance and fairly wide impedance bandwidth.

A large number of designs have been developed with remarkable results. However, there are still some nonnegligible drawbacks for most of the existing ME dipole antennas and dual-band antennas. ME antennas usually have complex and bulky structures $[5,6]$. In addition, the ME antennas which are made of special dielectric material or with differential feeding structure [8] are expensive. And the conventional dual-band antennas are usually arrayed in order to improve gains $[1,11]$, which have the same drawbacks as the existing ME antennas. Otherwise, they are single ones with low gains $[12,13]$. Therefore, a novel design of dual-band magnetoelectric dipole unidirectional antenna for $2 \mathrm{G} / 3 \mathrm{G} / \mathrm{LTE}$ applications with low profile structure, high gains, easy fabrication, and low cost, is highly desirable. Recently, a dual-wideband ME dipole antenna has been proposed for base stations [4]. However, it still cannot cover the whole LTE frequency band 


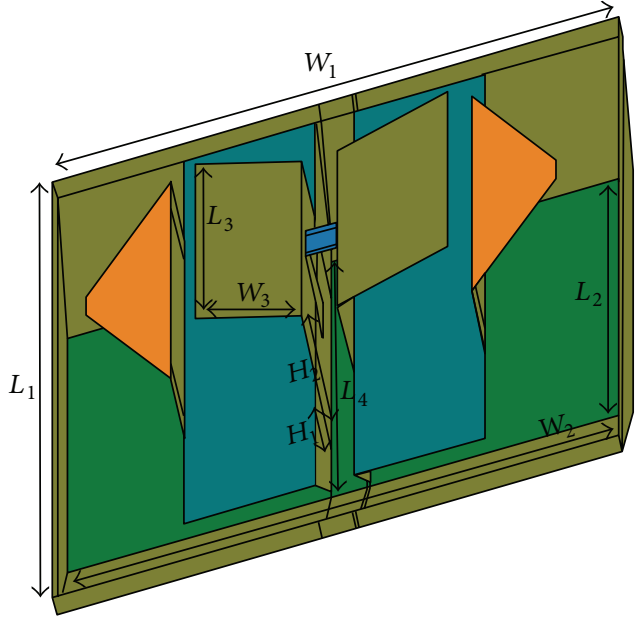

(a) $3 \mathrm{D}$ view

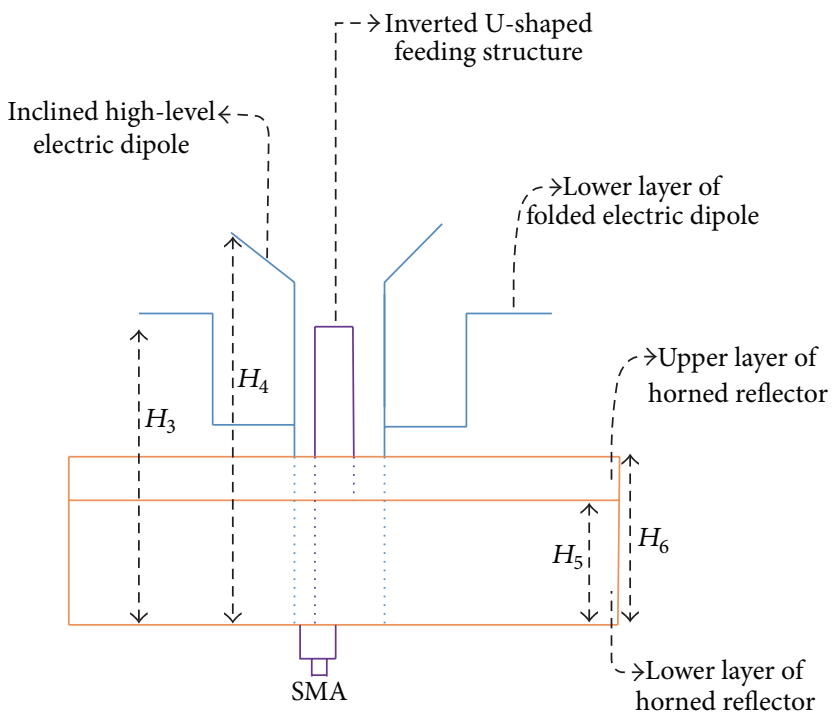

(c) Side view

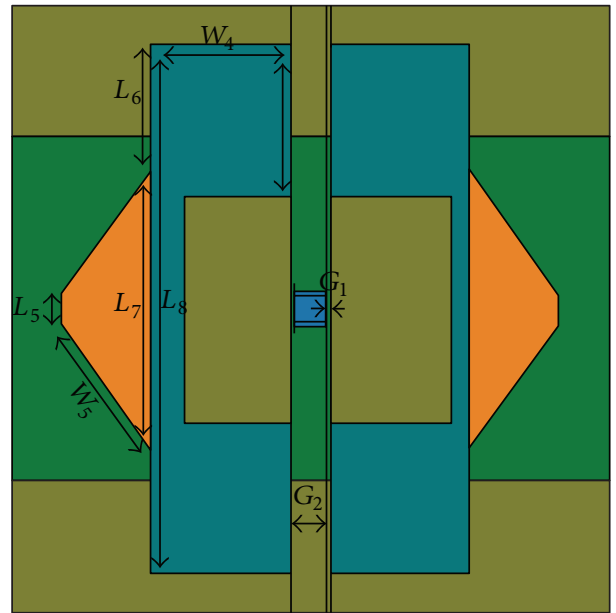

(b) Top view

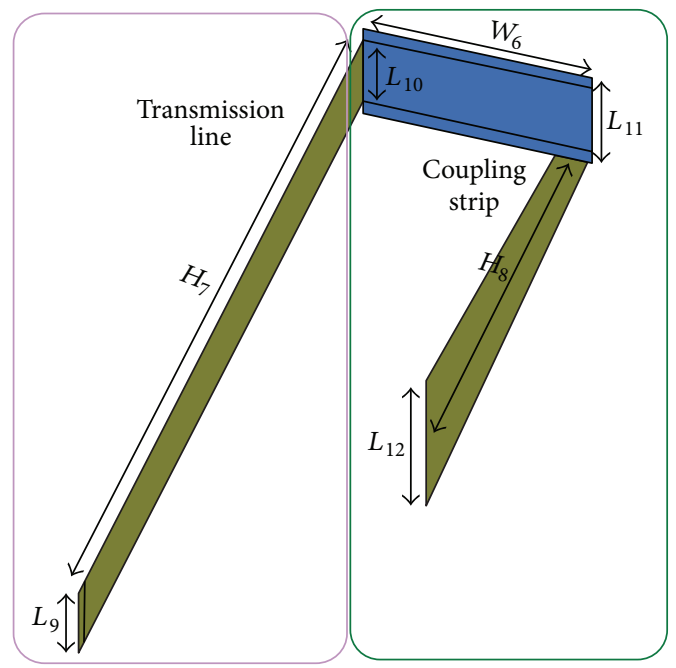

(d) Feeding structure

FIGURE 1: Geometry of the proposed antenna.

in cellular networks. Moreover, the gain drops sharply since it is ineffective in suppressing the radiation main beam which is split into two beams at high frequencies.

In this paper, instead of a horizontal planar electric dipole proposed in $[2,5,6]$ or notch slots as in [3], a double-layer folded electric dipole structure with a certain inclination is utilized to provide wideband nature by changing the current distribution. In addition, for further size reduction and better impedance matching, inverted U-shaped and tapered-line designs are adopted in the novel feeding structure. Finally, to improve the stable gains and symmetrical unidirectional radiation patterns, a modified horned reflector is employed. In comparison with the existing $\mathrm{ME}$ dipole antennas, it is completely made of copper and easy to fabricate at low cost. In order to analyze the antenna structure effectively, we first propose a simplified equivalent circuit of the double-layer ME dipole antenna model and then derive its equivalent formula to guide the design. Measurements and simulations are performed to verify the design. To the best of our knowledge, the proposed antenna is the first double-layer magnetoelectric dipole antenna.

\section{Antenna Description and Design Geometry}

Fundamentally, the proposed antenna consists of a doublelayer folded electric dipole, an inverted U-shaped taperedline feeding structure and a horned reflector. The dimensions of the antenna are $200 \times 140 \times 51 \mathrm{~mm}^{3}$. Besides, the thickness of the patches adopts $0.3 \mathrm{~mm}$ in view of the versatility and cost.

2.1. Double-Layer Folded Magnetoelectric Dipole Geometry. Figure 1 shows the geometry of the presented antenna. As 
shown in the 3D view and top view, the upper inclined rectangular patches and the lower horizontal rectangular patches are joined by upper vertical rectangular patches. The lower horizontal rectangular patches are connected to a pair of higher isosceles trapezoid patches by a pair of rectangular patches. Besides, the folded lower patches are designed to reduce the size and serve the lower frequency band while the inclined upper patches are devised to provide the upper frequency band. All of them compose the double-layer electric dipoles. In comparison with [3], the double-layer electric dipoles are more effective in increasing bandwidth at high frequencies. Figures $1(a)$ and $1(c)$ show that the lower horizontal rectangular patches are also connected to the ground plane by two lower vertical-isosceles trapezoid shorted patches which are separated by a gap of $G_{1}=13 \mathrm{~mm}$. The two lower vertical shorted patches and the portion of the ground plane between them form a magneto dipole, which is equivalent to a parallel resonant capacitor. The electric dipoles and the magneto dipole jointly constitute a ME dipole which is placed in the center of a horn-shaped reflector. Excellent complementary electrical characteristics are resulted from mutual coordination between them.

2.2. Inverted U-Shaped Tapered-Line Feeding Structure. In order to excite the antenna while reducing the size of the antenna and achieving good impedance matching, a novel inverted U-shaped tapered-line feeding structure is introduced. As shown in Figure 1(d), in comparison with [3], inverted U-shaped tapered-line feeding structure can easily attain better impedance matching, since tapered line can simply provide more degree of freedom for impedance matching. The feeding structure includes a transmission line and a coupling strip. The rectangular transmission line separates $1 \mathrm{~mm}$ from one of the vertical patches. It is vertically placed to the ground plane and the bottom end is connected to a SMA connector underneath the ground plane. The coupling strip consists of a horizontal rectangular patch and a vertical isosceles-trapezoid patch, the bottom of which is wider than the top. The horizontal part couples electrical energy to the antenna while the vertical part works together with one of the other vertical patches to compensate the inductance caused by the horizontal part. In comparison with the staircaseshaped feeding structure in $[3,5]$, the inverted L-shaped coupling strip incorporated with the trapezoidal microstrip are simple and easy for excellent impedance matching.

2.3. Double-Layer Horn-Shaped Reflector. Figures 1(a) and $1(c)$ show the geometry of the proposed double-layer horned reflector. At the upper layer, there is a rectangular box without upper surface and lower surface, whose dimensions are $200 \times$ $140 \times 5 \mathrm{~mm}^{3}$. And at the lower layer, there is a square horn whose upper surface and lower surface are $200 \times 140 \mathrm{~mm}^{2}$ and $200 \times 80 \mathrm{~mm}^{2}$, respectively; the distance between them is $15 \mathrm{~mm}$.

The detailed dimensions for the antenna are summarized in Table 1.
TABLE 1: Dimensions for the proposed antenna.

\begin{tabular}{|c|c|}
\hline Parameters & Values $/ \mathrm{mm}$ \\
\hline \multirow{4}{*}{$W 1$} & 200 \\
\hline & $\left(0.593 \lambda_{L}\right)$ \\
\hline & $\left(1.357 \lambda_{H}\right)$ \\
\hline & 200 \\
\hline \multirow[t]{3}{*}{$W 2$} & $\left(0.593 \lambda_{L}\right)$ \\
\hline & $\left(1.357 \lambda_{H}\right)$ \\
\hline & 36 \\
\hline \multirow[t]{3}{*}{ W3 } & $\left(0.107 \lambda_{L}\right)$ \\
\hline & $\left(0.244 \lambda_{H}\right)$ \\
\hline & 47 \\
\hline \multirow[t]{3}{*}{ W4 } & $\left(0.139 \lambda_{L}\right)$ \\
\hline & $\left(0.319 \lambda_{H}\right)$ \\
\hline & 42.4 \\
\hline \multirow[t]{3}{*}{ W5 } & $\left(0.126 \lambda_{L}\right)$ \\
\hline & $\left(0.288 \lambda_{H}\right)$ \\
\hline & 11 \\
\hline \multirow[t]{3}{*}{ W6 } & $\left(0.033 \lambda_{L}\right)$ \\
\hline & $\left(0.075 \lambda_{H}\right)$ \\
\hline & 140 \\
\hline \multirow[t]{3}{*}{$L 1$} & $\left(0.415 \lambda_{L}\right)$ \\
\hline & $\left(0.950 \lambda_{H}\right)$ \\
\hline & 80 \\
\hline \multirow[t]{3}{*}{$L 2$} & $\left(0.237 \lambda_{L}\right)$ \\
\hline & $\left(0.543 \lambda_{H}\right)$ \\
\hline & 52 \\
\hline \multirow[t]{3}{*}{$L 3$} & $\left(0.154 \lambda_{L}\right)$ \\
\hline & $\left(0.353 \lambda_{H}\right)$ \\
\hline & 78 \\
\hline \multirow[t]{3}{*}{$L 4$} & $\left(0.231 \lambda_{L}\right)$ \\
\hline & $\left(0.529 \lambda_{H}\right)$ \\
\hline & 6 \\
\hline \multirow[t]{3}{*}{ L5 } & $\left(0.018 \lambda_{L}\right)$ \\
\hline & $\left(0.041 \lambda_{H}\right)$ \\
\hline & 28 \\
\hline \multirow[t]{3}{*}{ L6 } & $\left(0.083 \lambda_{L}\right)$ \\
\hline & $\left(0.190 \lambda_{H}\right)$ \\
\hline & 66 \\
\hline \multirow[t]{3}{*}{$L 7$} & $\left(0.196 \lambda_{L}\right)$ \\
\hline & $\left(0.448 \lambda_{H}\right)$ \\
\hline & 122 \\
\hline \multirow[t]{3}{*}{ L8 } & $\left(0.362 \lambda_{L}\right)$ \\
\hline & $\left(0.828 \lambda_{H}\right)$ \\
\hline & 6 \\
\hline \multirow[t]{3}{*}{$L 9$} & $\left(0.018 \lambda_{L}\right)$ \\
\hline & $\left(0.041 \lambda_{H}\right)$ \\
\hline & 6 \\
\hline \multirow[t]{3}{*}{$L 10$} & $\left(0.018 \lambda_{L}\right)$ \\
\hline & $\left(0.041 \lambda_{H}\right)$ \\
\hline & 8 \\
\hline \multirow[t]{2}{*}{$L 11$} & $\left(0.024 \lambda_{L}\right)$ \\
\hline & $\left(0.054 \lambda_{H}\right)$ \\
\hline
\end{tabular}


TABLE 1: Continued.

\begin{tabular}{|c|c|}
\hline Parameters & Values/mm \\
\hline & 12 \\
\hline \multirow[t]{3}{*}{$L 12$} & $\left(0.036 \lambda_{L}\right)$ \\
\hline & $\left(0.081 \lambda_{H}\right)$ \\
\hline & 23 \\
\hline \multirow[t]{3}{*}{$H 1$} & $\left(0.068 \lambda_{L}\right)$ \\
\hline & $\left(0.156 \lambda_{H}\right)$ \\
\hline & 43 \\
\hline \multirow[t]{3}{*}{$H 2$} & $\left(0.128 \lambda_{L}\right)$ \\
\hline & $\left(0.292 \lambda_{H}\right)$ \\
\hline & 42 \\
\hline \multirow[t]{3}{*}{$H 3$} & $\left(0.125 \lambda_{L}\right)$ \\
\hline & $\left(0.285 \lambda_{H}\right)$ \\
\hline & 51 \\
\hline \multirow[t]{3}{*}{$H 4$} & $\left(0.151 \lambda_{L}\right)$ \\
\hline & $\left(0.346 \lambda_{H}\right)$ \\
\hline & 15 \\
\hline \multirow[t]{3}{*}{ H5 } & $\left(0.044 \lambda_{L}\right)$ \\
\hline & $\left(0.102 \lambda_{H}\right)$ \\
\hline & 20 \\
\hline \multirow[t]{3}{*}{ H6 } & $\left(0.059 \lambda_{L}\right)$ \\
\hline & $\left(0.136 \lambda_{H}\right)$ \\
\hline & 41 \\
\hline \multirow[t]{3}{*}{$H 7$} & $\left(0.122 \lambda_{L}\right)$ \\
\hline & $\left(0.278 \lambda_{H}\right)$ \\
\hline & 24 \\
\hline \multirow[t]{3}{*}{ H8 } & $\left(0.071 \lambda_{L}\right)$ \\
\hline & $\left(0.163 \lambda_{H}\right)$ \\
\hline & 1 \\
\hline \multirow[t]{3}{*}{$G 1$} & $\left(0.003 \lambda_{L}\right)$ \\
\hline & $\left(0.007 \lambda_{H}\right)$ \\
\hline & 13 \\
\hline \multirow[t]{2}{*}{ G2 } & $\left(0.039 \lambda_{L}\right)$ \\
\hline & $\left(0.088 \lambda_{H}\right)$ \\
\hline
\end{tabular}

$\lambda_{L}$ is the center frequency of the lower frequency band, while $\lambda_{H}$ is the center frequency of the upper frequency band.

\section{Theory and Parametric Study}

Figures 2 and 3 show the schematic diagram and equivalentcircuit diagram of a simplified double-layer ME dipole antenna, respectively. According to the structure and function of the ME dipole antenna described in [14], the input impedance of antenna $Z_{\text {in }}$ can be expressed as follows:

$$
\begin{aligned}
Z_{\text {in }}= & Z_{o}+\left[Z_{L}(B D) \| Z_{C}(\text { Magnetic dipole })\right] \\
& +Z_{C}(B C+D E)+Z_{L}(\text { Electric dipoles }),
\end{aligned}
$$

where $Z_{o}, Z_{L}(B D), Z_{C}$ (magnetic dipole), $Z_{C}(B C+D E)$, and $Z_{L}$ (electric dipoles) are the characteristic impedance of transmission line, the impedance of horizontal part of coupling strip, the impedance of magnetic dipole, the impedance of capacitance between $B$ and $C, D$ and $E$, and the impedance of electric dipoles, respectively. In the resonant process, the horizontal part of coupling strip and electric dipoles mainly

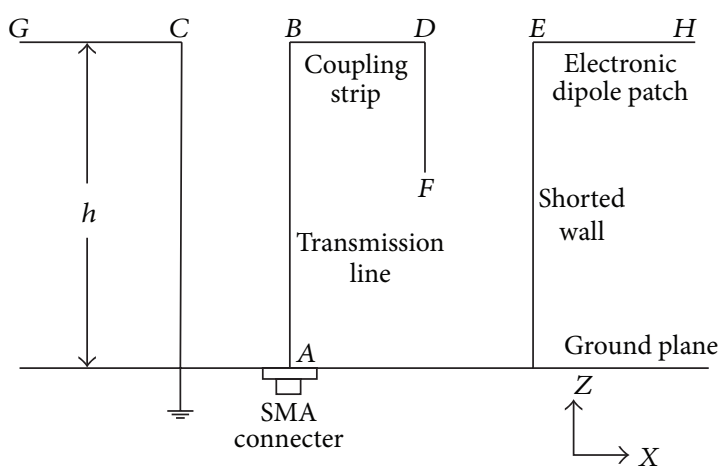

FIGURE 2: Schematic diagram of ME dipole antenna.

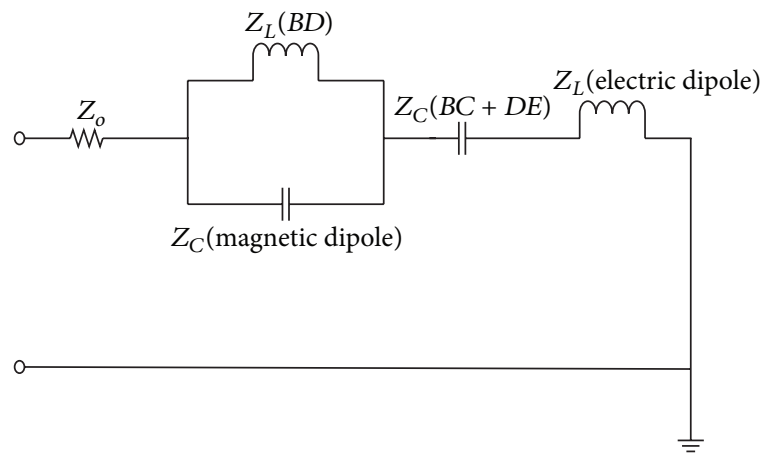

FIgURE 3: Equivalent circuit of ME dipole antenna.

show inductive while magnetic dipole, vertical portions of feeding structure, and shorted wall chiefly display capacitive.

Based on (1), it can be easily seen that the input impedance is mainly affected by the parameters of the horizontal length and vertical height of electric dipole, the horizontal length and vertical length of the coupling strip, and so on.

As stated above, in order to observe how the dimensions of the antenna affect the performances, a parametric study is carried out by electromagnetic simulation software $I E 3 D$ [15]. Throughout the course of the study, the metallic layers are set to have $0.3 \mathrm{~mm}$ thickness for practical applications. When one parameter is studied, the others are unchangeable. To obtain stable gain and excellent impedance matching, some important parameters of the U-shaped bow-tie ME dipole, inverted U-shaped tapered-line feeding structure, and horn-shaped reflector are selected to be studied.

\subsection{Effect of the Double-Layer Electric Dipole Structure}

3.1.1. The Inclination of the Upper Electric Dipole. To reveal the impact of the inclination of the upper electric dipole, the height of $\mathrm{H}_{4}$ is investigated while keeping the length of $\mathrm{H}_{2}=$ $43 \mathrm{~mm}$ constant. The heights of $H_{4}$ at 43, 51, and $59 \mathrm{~mm}$ are compared in the aspects of SWR and gain.

As shown in Figure 4, at the lower frequency band, the SWR of the proposed antenna with $H_{4}=43 \mathrm{~mm}$ rises sharply and the gain drops correspondingly, while the other are $\left(\mathrm{H}_{4}=\right.$ $51 \mathrm{~mm}, H_{4}=59 \mathrm{~mm}$ ) have no obvious fluctuation. And at 


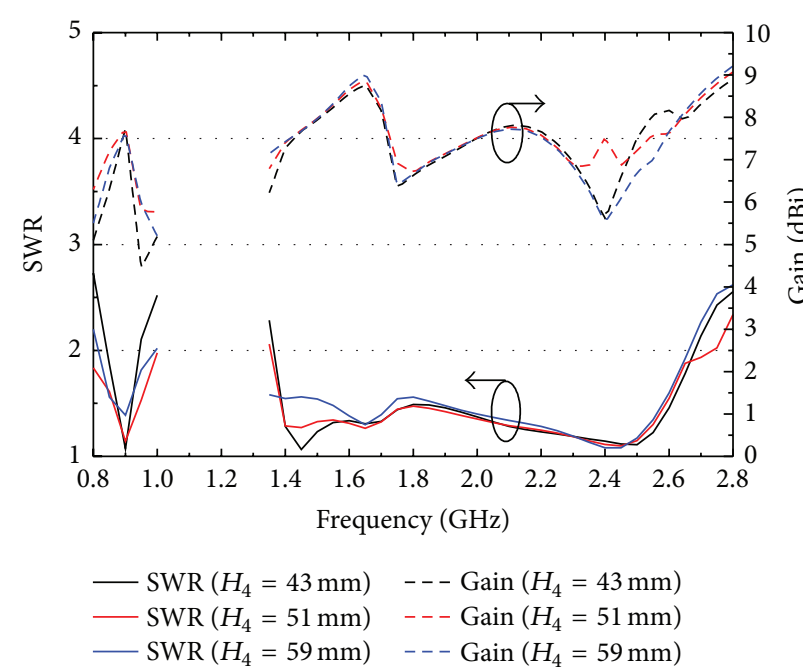

Figure 4: Effects of the height of $H_{4}$.

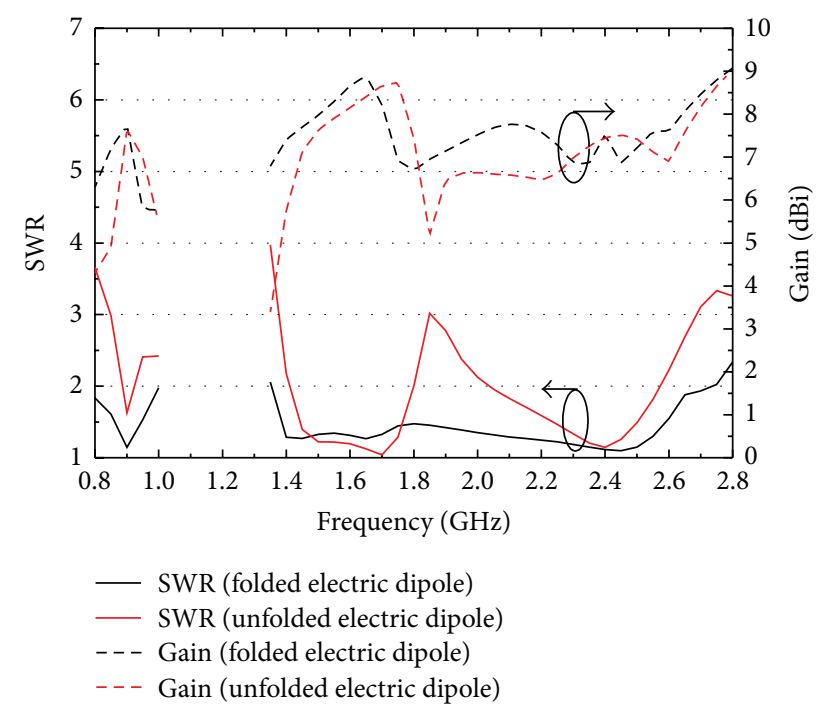

FIGURE 5: Effects of folded and unfolded electric dipoles.

the high frequencies of the upper frequency band, the gain of the proposed antenna with $H_{4}=51 \mathrm{~mm}$ is more stable than the other with changes in the range of $2 \mathrm{dBi}$. At the other part of the frequency bands, the SWRs and the gains of the antennas with the different dimensions keep stable. This is because the upper electric dipole with a suitable inclination is better on impedance matching and radiation patterns at high frequencies. Hence, the proposed electric dipole with $\mathrm{H}_{4}=$ $51 \mathrm{~mm}$ can broaden the impedance bandwidth significantly and keep gain stable.

3.1.2. The Low-Level Folded Electric Dipole. To depict effect of the folded electric dipoles, SWRs and gains of the folded electric dipoles and unfolded electric dipoles are compared, as shown in Figure 5. It is clearly seen that there are obvious fluctuations in the SWR and gains of the unfolded electric dipoles at low and middle frequencies due to impedance

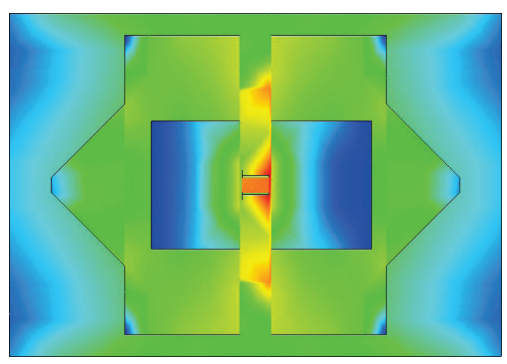

(a) $0.9 \mathrm{GHz}$

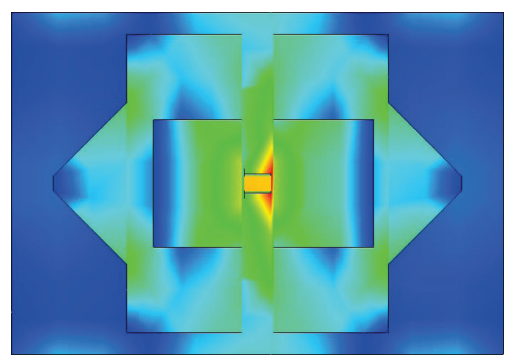

(b) $1.8 \mathrm{GHz}$

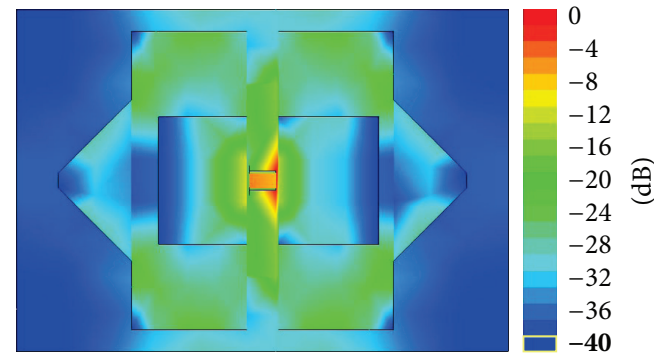

(c) $2.7 \mathrm{GHz}$

Figure 6: Current distributions on the electric dipole.

mismatching. As a result, the impedance bandwidth becomes narrow. Thus, folded dipoles can provide more flexible impedance matching in a limited space.

Besides, to depict the working mechanism of the doublelayer electric dipoles, the simulations of surface current distributions at $0.9 \mathrm{GHz}, 1.8 \mathrm{GHz}$, and $2.7 \mathrm{GHz}$ are carried out, as shown in Figure 6.

It is clearly seen that the current distribution at $0.9 \mathrm{GHz}$ demonstrates much stronger currents around the low-level electric dipoles than the high-level ones. However, the current distributions on the high-level electric dipoles significantly enhance as the frequency increases. In other words, the folded low-level electric dipoles mainly serve the lower frequency band while the inclined high-level electric dipoles work for the upper frequency band.

3.2. Effect of the Inverted U-Shaped Tapered-Line Feeding Structure. As mentioned above, in reality, the feeding strip can be divided into two parts: the transmission line and the coupling strip. The coupling strip is a critical part to bandwidth and gain of the antenna.

3.2.1. The Effect of the Horizontal Portion of Coupling Strip. In order to reveal the impact of the horizontal portion of 


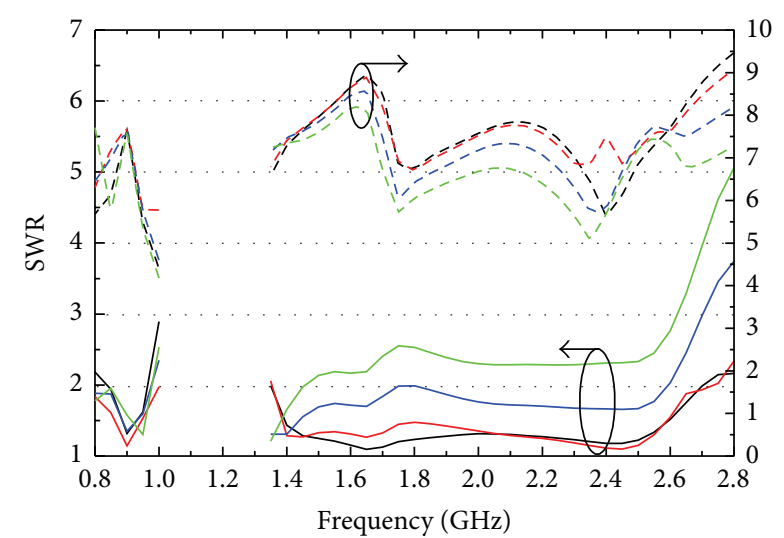

$$
\begin{array}{ll}
-\operatorname{SWR}\left(W_{6}=7 \mathrm{~mm}\right) & --\operatorname{Gain}\left(W_{6}=7 \mathrm{~mm}\right) \\
\operatorname{SWR}\left(W_{6}=11 \mathrm{~mm}\right) & ---\operatorname{Gain}\left(W_{6}=11 \mathrm{~mm}\right) \\
-\operatorname{SWR}\left(W_{6}=15 \mathrm{~mm}\right) & ---\operatorname{Gain}\left(W_{6}=15 \mathrm{~mm}\right) \\
\operatorname{SWR}\left(W_{6}=19 \mathrm{~mm}\right) & ---\operatorname{Gain}\left(W_{6}=19 \mathrm{~mm}\right)
\end{array}
$$

Figure 7: Effects of the $W_{6}$ while keeping $L_{11}=8 \mathrm{~mm}$.

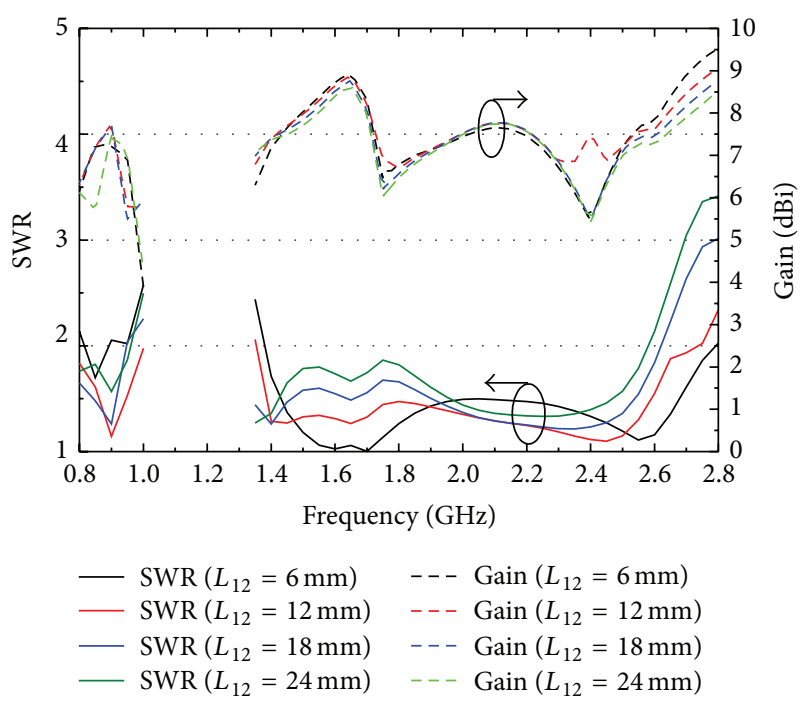

FIGURE 8: Effects of the $L_{12}$ while keeping $H_{8}=24 \mathrm{~mm}$.

the strip, we study the length of $W_{6}$ while keeping the width of $L_{11}=8 \mathrm{~mm}$ constant for good performance. It can be found that the gains are very sensitive to the parameters. As shown in Figure 7 , a shorter $W_{6}$ brings a larger fluctuation at low frequencies while a longer $W_{6}$ produces a more obvious variation at high frequencies. Correspondingly, there are significant variations with the gains when the length of $W_{6}$ is too short or long. Thus, $W_{6}=11 \mathrm{~mm}$ and $L_{11}=8 \mathrm{~mm}$ can be selected for wider bandwidth and more stable gain. The effect of the width of the horizontal portion of the strip $\left(L_{10}\right)$ is similar to $W_{6}$. In brief, it can be concluded that the horizontal portion of the strip is crucial for wide bandwidth and stable gain. And it is equivalent to an inductive impedance which corresponds to $Z_{L}$ (electric dipoles) of the equivalent circuit in Figure 3.
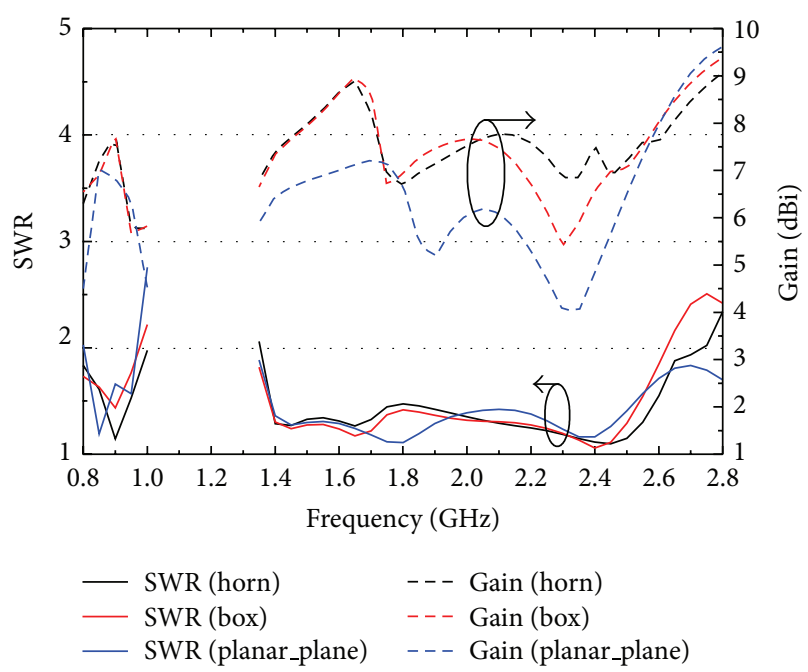

FIGURE 9: Effects of the reflectors.

3.2.2. The Effect of the Vertical Portion of Coupling Strip and Magnetic Dipole. The vertical portion of the coupling strip coordinated with the vertically oriented patch of magnetic dipole to compensate the inductance caused by the horizontal portion [5]. To investigate the usefulness of the vertical portion of the coupling strip, we studied its width $\left(L_{12}\right)$ in the case of $H_{8}=24 \mathrm{~mm}$. As shown in Figure 8, in the upper frequency band, there is no obvious variation when $L_{12}$ changes. However, either a shorter or a longer $L_{12}$ brings out a larger SWR variation and unstable gain at lower frequency band. The impedance bandwidth becomes narrow when $L_{12}$ is not equal to $12 \mathrm{~mm}$, due to impedance mismatching. Thus, $L_{12}=12 \mathrm{~mm}$ is chosen for stable gain and low SWR. The impedance between the vertical portion of the coupling strip and the shorted wall is equivalent to a series capacitor $Z_{C}(B C)$ in Figure 3.

3.3. Effect of the Horn-Shaped Reflector. Figure 9 shows the effects on simulated gains and SWRs in the conditions of adopting a double-layer horned reflector, a box-shaped reflector, or a planar reflector. Among them, the fence heights $\left(\mathrm{H}_{6}\right)$ of the box-shaped and horned reflectors are the same. It can be easily seen that, with a horned reflector, the gain is more stable and the bandwidth is much wider than the other are (box-shaped reflector and planar reflector). In addition, with a planar reflector, the gain is the lowest.

As shown in Figure 10, in comparison with radiation patterns, the proposed antenna with a double-layer horned reflector has lower cross-polarization than the one with a planar reflector at high frequencies. Besides, the front-to-back ratio of the proposed antenna with a double-layer horned reflector is higher than the other. The reason for this is that adopting the double-layer horned reflector is more effective in suppressing the radiation main beam which divides into two beams at high frequencies. Therefore, it is an effective way to improve broadside gain and impedance matching with the double-layer horned reflector. 


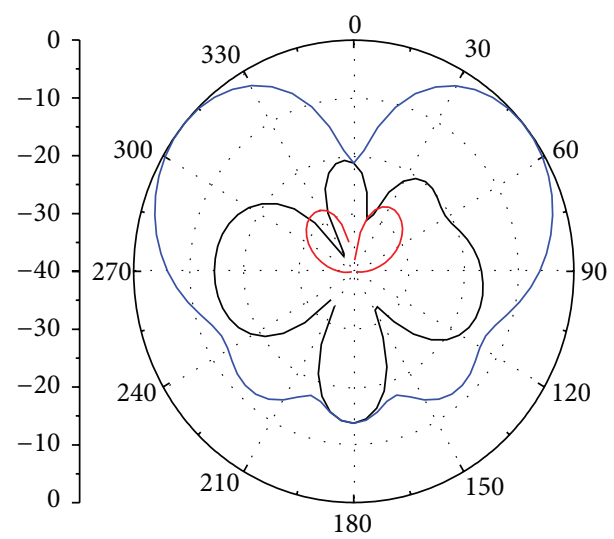

(a) With a planar reflector

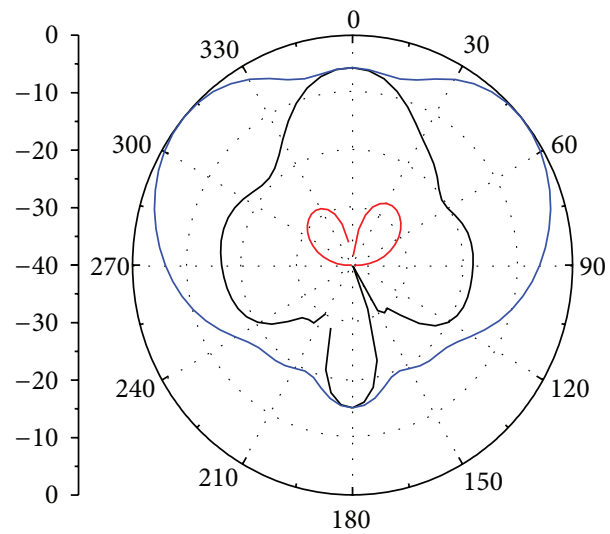

(b) With a box reflector

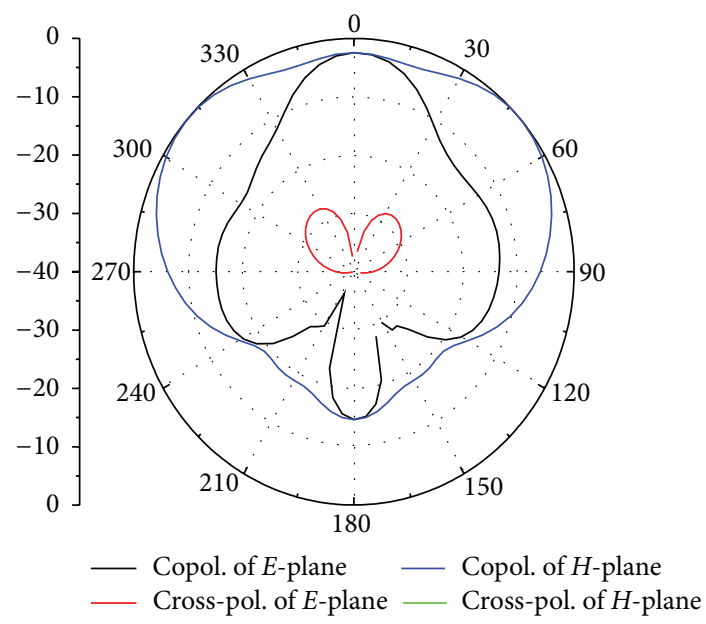

(c) With a horn reflector

Figure 10: Effects of the reflectors (radiation patterns at $2.7 \mathrm{GHz}$ ).

\section{Experimental Results}

To verify the proposed design, an antenna prototype according to the dimensions summarized in Table 1 was constructed, as shown in Figure 11. Measured results of SWR, antenna gain, and radiation patterns were obtained by Agilent E5071C network analyzer and a SATIMO antenna measurement system.

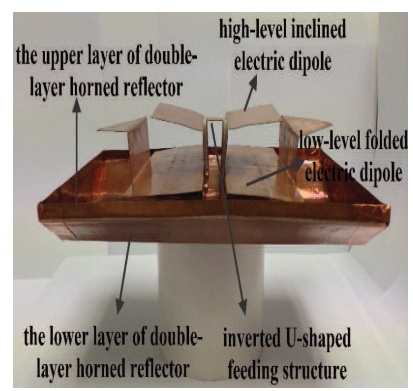

(a) Front side view

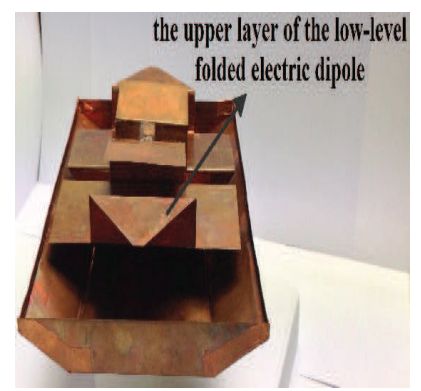

(b) Left side view

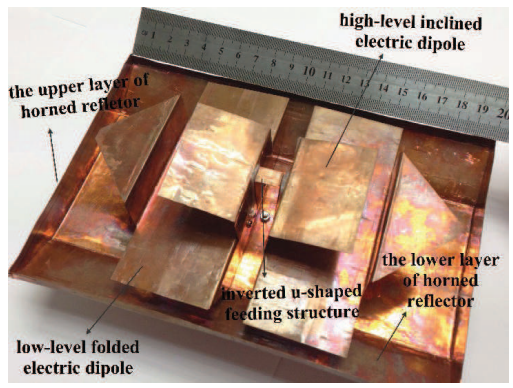

(c) 3D view

FIGURE 11: Photos of the proposed antenna.

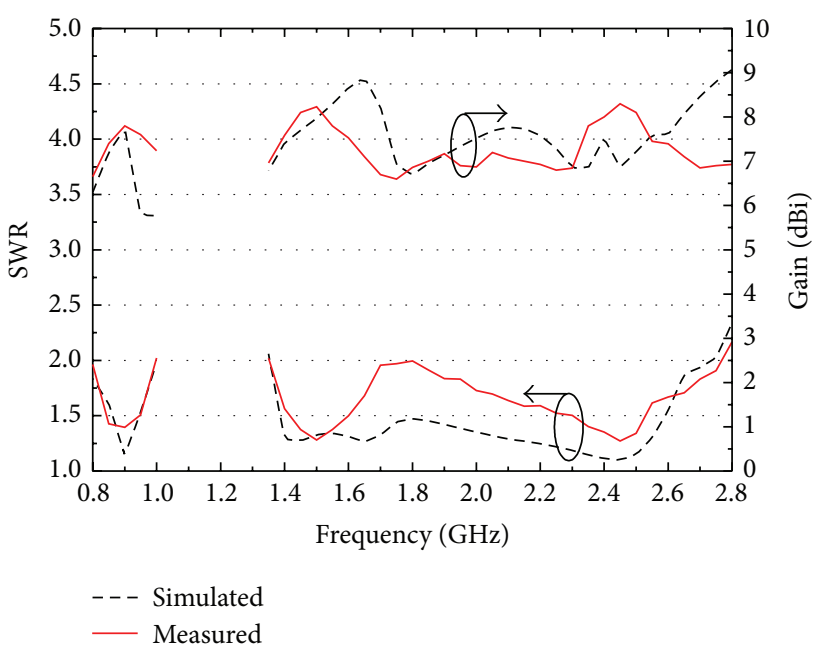

FIGURE 12: Simulated and measured SWRs and broadside gains.

\section{SWR and Gain}

Figure 12 shows good agreement between the simulated and measured SWRs and gains of the proposed antenna. It is easily observed that, for the lower frequency band, the simulated impedance bandwidth is $26.7 \%$ from 0.78 to $1.02 \mathrm{GHz}$, while the measured impedance bandwidth is $24.4 \%$ from 0.79 to $1.01 \mathrm{GHz}$ (SWR $\leq 2$ ). For the upper frequency band, the simulated impedance bandwidth is $68.3 \%$ from 1.35 to $2.75 \mathrm{GHz}$, while the measured impedance bandwidth is $67.3 \%$ from 1.38 to $2.78 \mathrm{GHz}$ (SWR $\leq 2$ ). Both of them cover the frequency bands of $2 \mathrm{G} / 3 \mathrm{G} / \mathrm{LTE}(4 \mathrm{G})$ systems. The measured gain varies between $6.6 \mathrm{dBi}$ and $7.8 \mathrm{dBi}$ for the lower band, while the measured antenna gain varies between $6.6 \mathrm{dBi}$ and $8.3 \mathrm{dBi}$ for 


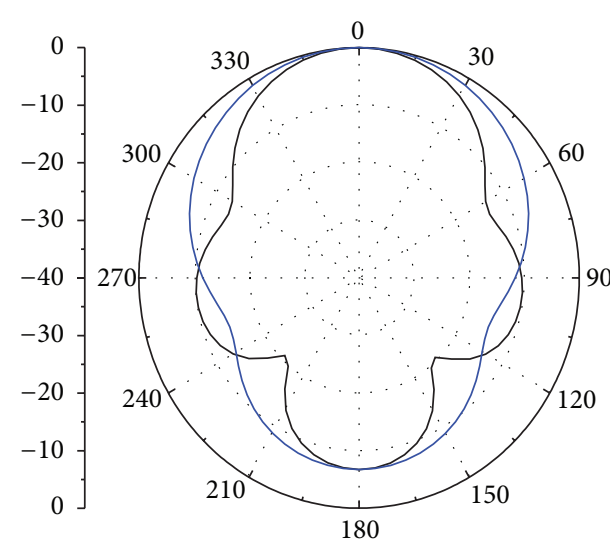

(a) $0.9 \mathrm{GHz}$ (Simulated)

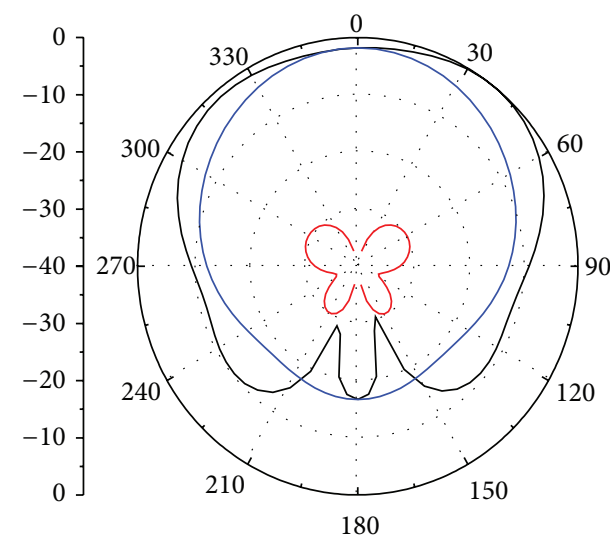

(c) $1.8 \mathrm{GHz}$ (Simulated)

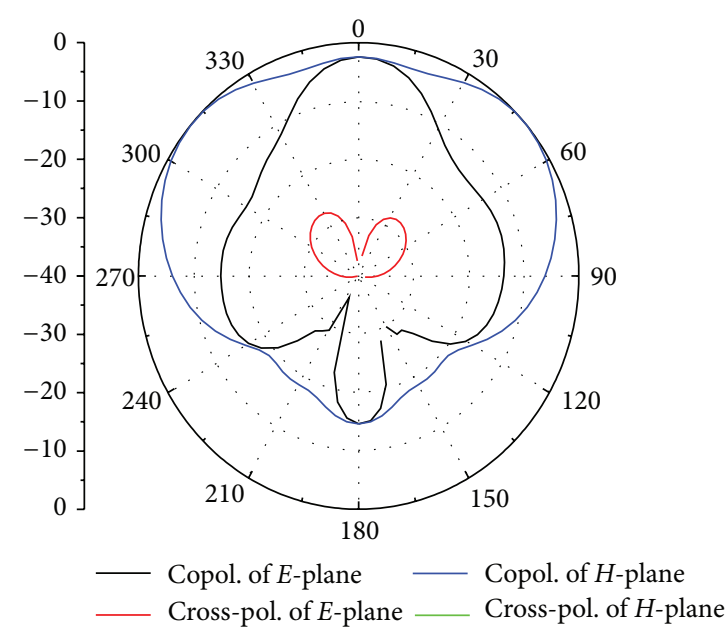

(e) $2.7 \mathrm{GHz}$ (Simulated)

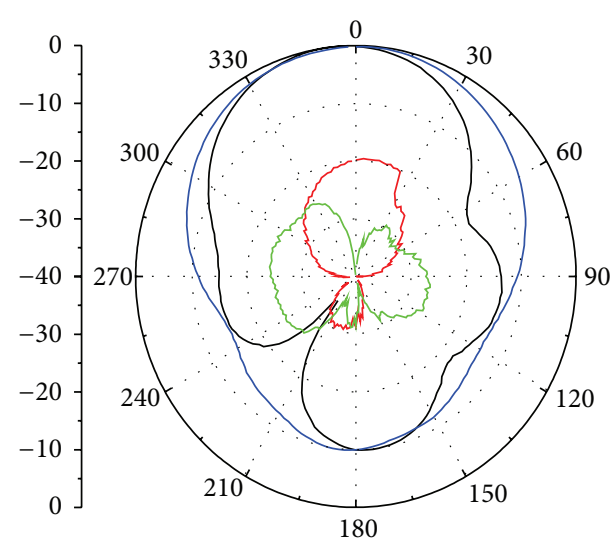

(b) $0.9 \mathrm{GHz}$ (Measured)

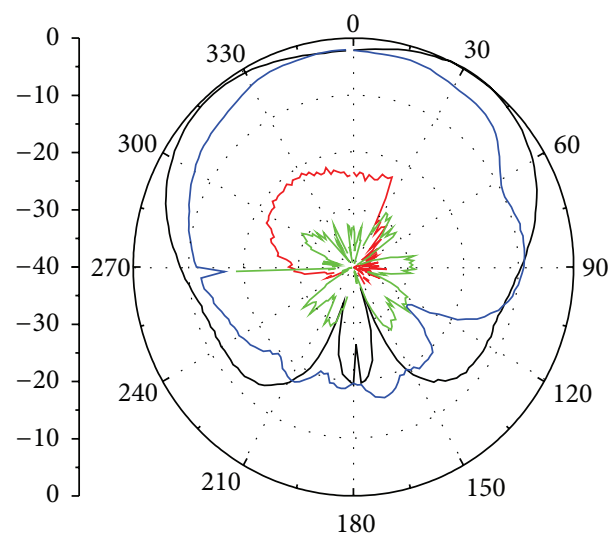

(d) $1.8 \mathrm{GHz}$ (Measured)

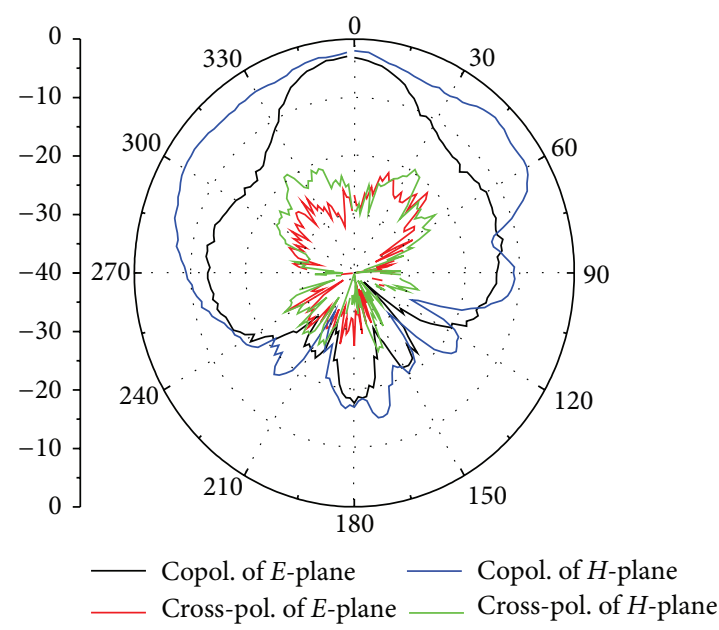

(f) $2.7 \mathrm{GHz}$ (Measured)

FIGURE 13: Simulated and measured radiation patterns at $0.9 \mathrm{GHz}, 1.8 \mathrm{GHz}$, and $2.7 \mathrm{GHz}$.

the upper band. In addition, the absolute bandwidth at upper band is wider than previous dual-band ME dipole antenna [4] by $34.6 \%$, which is also completely made of copper.

5.1. Radiation Patterns. The simulated and measured radiation patterns at frequencies of $0.9 \mathrm{GHz}, 1.8 \mathrm{GHz}$, and $2.7 \mathrm{GHz}$ are shown in Figure 13, respectively. Well agreement is achieved. The antenna displays good unidirectional radiation patterns. Over the operating frequency band, nearly equal and symmetric radiation patterns in the $E$ - and $H$-planes are achieved, and the main beams are fixed to the broadside direction. In addition, the measured front-to-back ratios are above $10 \mathrm{~dB}$, and the cross polarization levels in the $E$ - and $H$-planes are both below $-22 \mathrm{~dB}$. (Since the cross-polarized radiation levels in $E$ - and $H$-plane are lower than $-40 \mathrm{~dB}$, they cannot be seen in some graphs.) 


\section{Conclusion}

A novel dual-wideband double-layer magnetoelectric dipole unidirectional antenna with a modified horned reflector for $2 \mathrm{G} / 3 \mathrm{G} / \mathrm{LTE}$ applications is proposed. The design has been verified through measurements on a prototype of the antenna. The results show good performance in terms of wider bandwidth and stable gain, which are $24.4 \%$ $(790 \mathrm{MHz}-1010 \mathrm{MHz})$ and $7.2 \pm 0.6 \mathrm{dBi}$ for the lower band $67.3 \%(1.38 \mathrm{GHz}-2.78 \mathrm{GHz})$ and $7.5 \pm 0.8 \mathrm{dBi}$ for the upper band. Besides, both simulated and measured results show low cross polarization, low back radiation, and symmetrical $E$ - and $H$-plane patterns. Moreover, compared with the existing ME dipole antennas, the proposed antenna which is completely made of copper can be easily fabricated at low cost and hence is suitable for $2 \mathrm{G} / 3 \mathrm{G} / \mathrm{LTE}$ systems.

However, due to the inherent limitation of threedimensional ME dipole antenna, its size is still somewhat large. In the future, if the ME dipole antenna can be further miniaturized without sacrificing performance, it would be more applicable for the portable devices.

\section{Acknowledgments}

The research was supported by grants from National Natural Science Foundation of China (no. 61139001) and the National Science and Technology Major Project (no. 2012ZX03003006).

\section{References}

[1] Y. H. Cui, R. L. Li, and P. Wang, "Novel dual-broadband planar antenna and its array for 2G/3G/LTE base stations," IEEE Transactions on Antennas and Propagation, vol. 61, no. 3, pp. 1132-1139, 2013.

[2] B. Q. Wu and K. M. Luk, "A magneto-electric dipole with a modified ground plane," IEEE Antennas and Wireless Propagation Letters, vol. 8, pp. 627-629, 2009.

[3] W. X. An, K. L. Lau, S. F. Li, and Q. Xue, "Wideband E-shaped dipole antenna with staircase-shaped feeding strip," Electronics Letters, vol. 46, no. 24, pp. 1583-1584, 2010.

[4] W. X. An, H. Wong, K. L. Lau, S. F. Li, and Q. Xue, "Design of broadband dual-band dipole for base station antenna," IEEE Transactions on Antennas and Propagation, vol. 60, no. 3, pp. 1592-1595, 2012.

[5] L. Ge and K. M. Luk, "A wideband magneto-electric dipole antenna," IEEE Transactions on Antennas and Propagation, vol. 60, no. 11, pp. 4987-4991, 2012.

[6] L. Ge and K. M. Luk, "A low-profile magneto-electric dipole antenna," IEEE Transactions on Antennas and Propagation, vol. 60, no. 4, pp. 1684-1689, 2012.

[7] Q. Xue, S. W. Liao, and J. H. Xu, "A differentially-driven dualpolarized magneto-electric dipole antenna," IEEE Transactions on Antennas and Propagation, vol. 61, no. 1, pp. 425-430, 2012.

[8] M. Li and K. M. Luk, "A differential-fed magneto-electric dipole antenna for ultra-wideband applications," in Proceedings of the International Symposium on Antennas and Propagation and USNC/URSI National Radio Science Meeting, (APSURSI '11), pp. 1482-1485, IEEE, Spokane, Wash, USA, July 2011.
[9] C. Tang and Q. Xue, "Vertical planar printed unidirectional antenna," IEEE Antennas and Wireless Propagation Letters, vol. 12, pp. 368-371, 2013.

[10] K. B. Ng, H. Wong, K. K. So, C. H. Chan, and K. M. Luk, " $60 \mathrm{GHz}$ plated through hole printed magneto-electric dipole antenna," IEEE Transactions on Antennas and Propagation, vol. 60, no. 7, pp. 3129-3136, 2012.

[11] J. H. Lu and S. W. You, "Novel dual-band design of planar dipole array for 4G LTE/WiMAX access points," International Journal of Antennas and Propagation, vol. 2011, Article ID 638034, 4 pages, 2011.

[12] J. Zhang, J. Ouyang, K. Z. Zhang, and F. Yang, "A novel dual-band MIMO antenna with lower correlation coefficient," International Journal of Antennas and Propagation, vol. 2012, Article ID 512975, 7 pages, 2012.

[13] B. Li and J. S. Hong, "Design of two novel dual band-notched UWB antennas," International Journal of Antennas and Propagation, vol. 2012, Article ID 303264, 7 pages, 2012.

[14] K. M. Luk and H. Wong, "A new wideband unidirectional antenna element," International Journal of Antennas and Propagation, vol. 1, no. 1, pp. 35-44, 2006.

[15] “IE3D," version 12.30, Zeland Software Inc., Fremont, Calif, USA, 2007. 

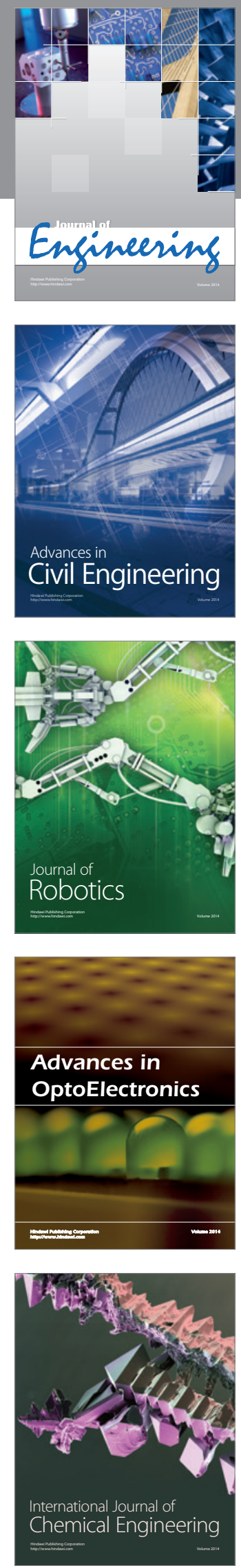

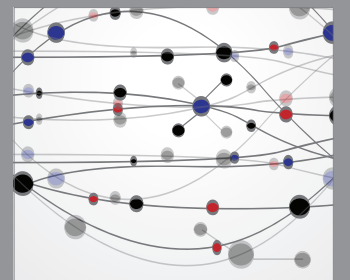

The Scientific World Journal
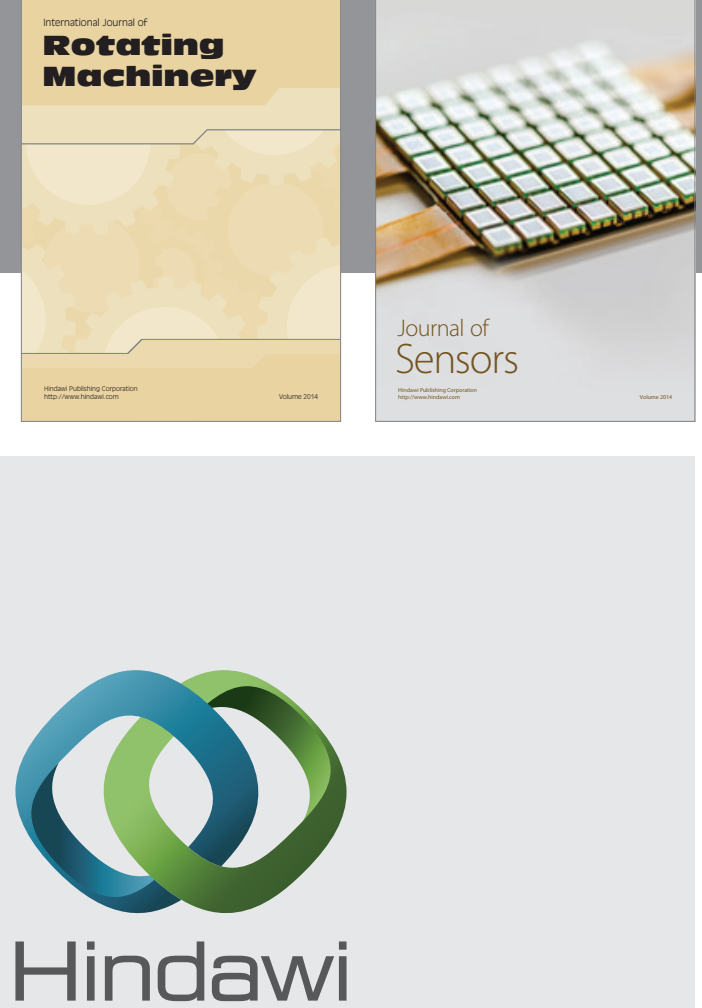

Submit your manuscripts at http://www.hindawi.com
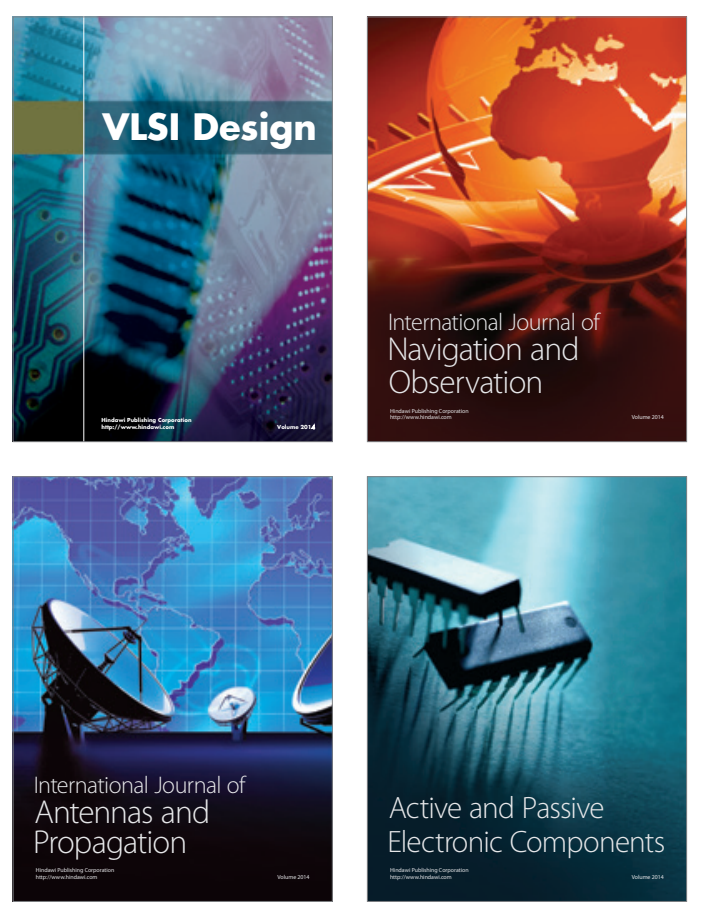
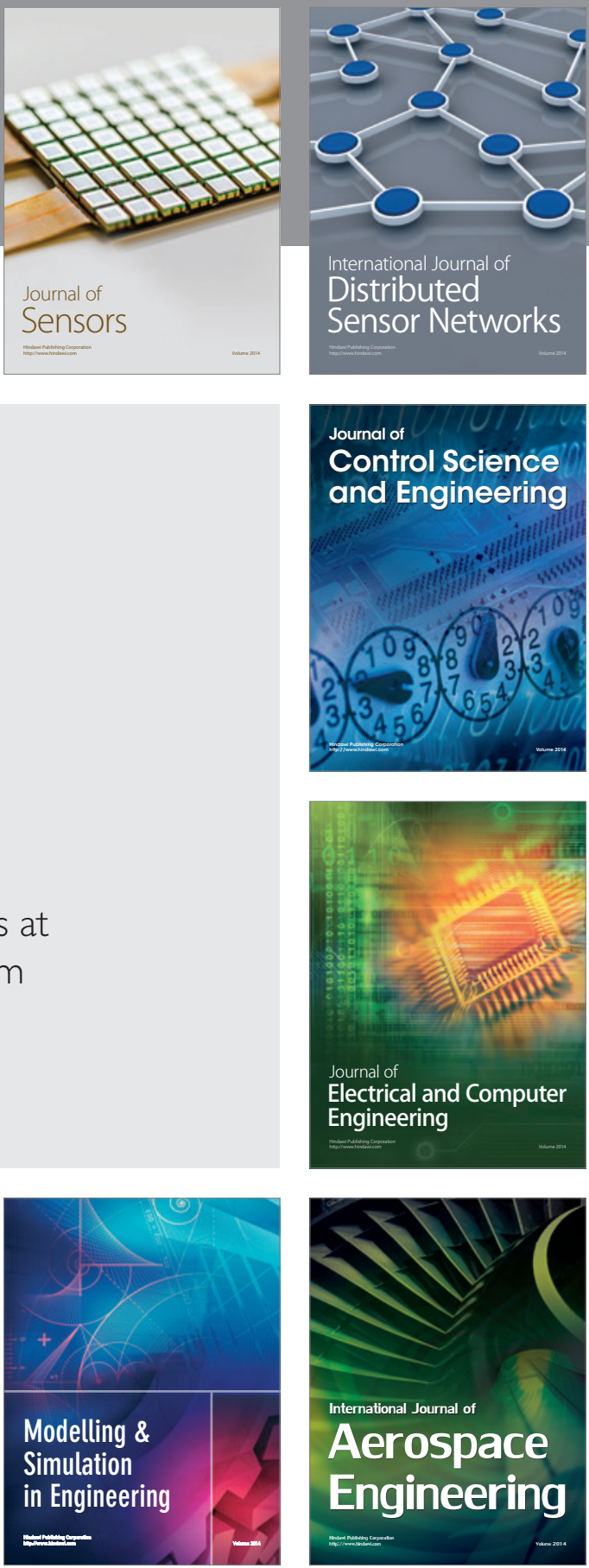

Journal of

Control Science

and Engineering
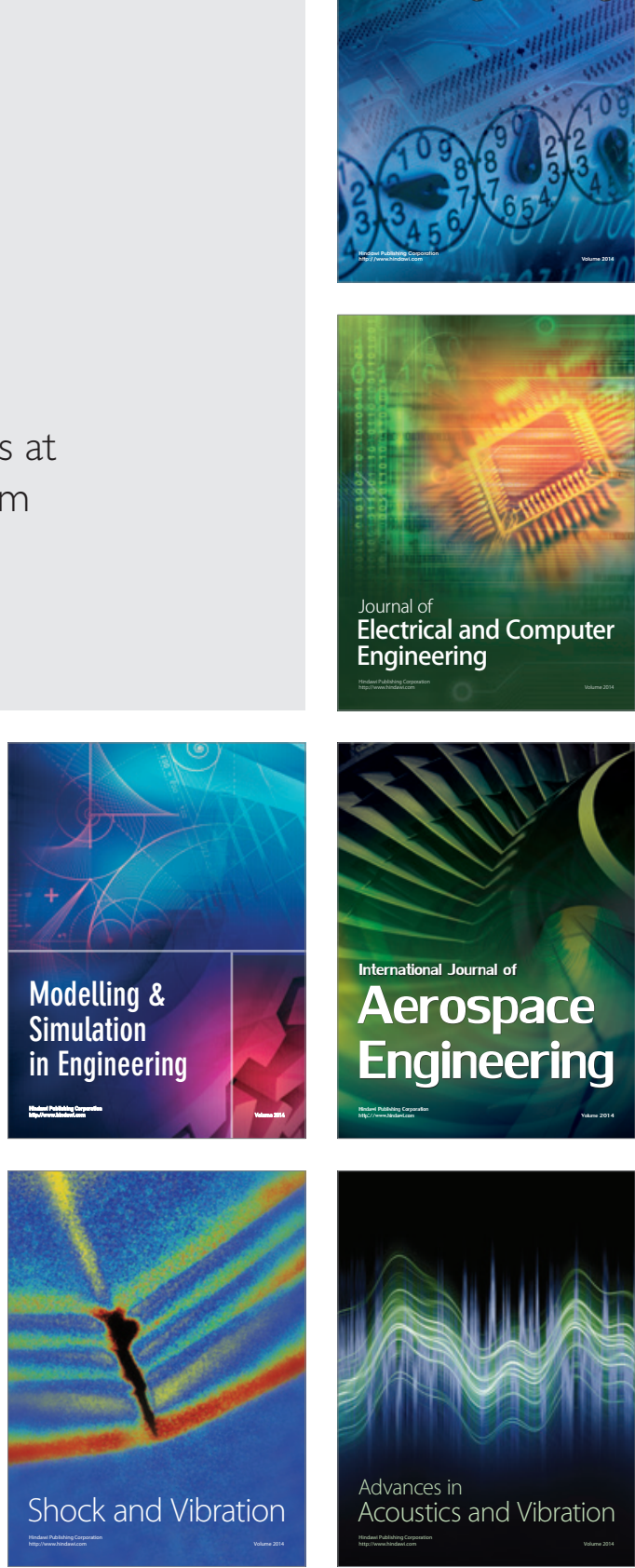